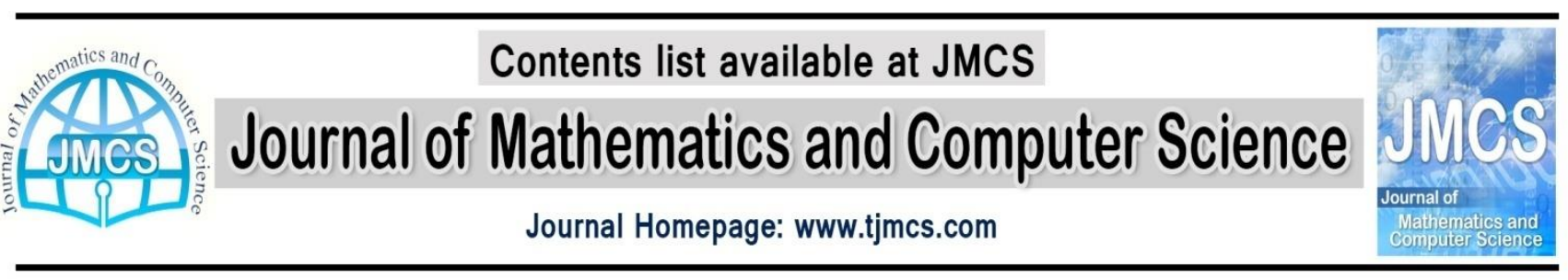

\title{
Product and Coproduct in the Category of Fuzzy Frames
}

\author{
Maryam Yaghoobi \\ Assistant Professor, Department of Mathematics, Payame Noor University, \\ P.O. Box 19395-3697, I.R.of Iran. \\ yaghoobim@yahoo.com
}

Article history:

Received June 2014

Accepted August 2014

Available online January 2015

\begin{abstract}
Frame theory is Lattice theory applied to topology. This approach to topology takes the lattices of open sets as the basic notion-it is "point free topology". There, one investigates typical properties of lattices of open sets that can be expressed without reference to points.

In this paper we generalise the concept of frame into a fuzzy frame. The category FFrm of fuzzy frame and fuzzy frame homomorphism is defined and we show that there exist products and coproducts in the category FFrm and to construct them explicitly and we conclude that the category FFrm is complete and cocomplete.
\end{abstract}

Keywords: Frame, Fuzzy frame ,Product, Coproduct,complete,cocomplete.

AMS Subject Classification :06D22,06D72,54A40.

\section{Introduction}

The first mathematician to take the notion of open set as basic to the study of continuity properties was Hausdorff in 1914. Using the lattice of open sets , Marshal stone[11] was able to give topological representation of Boolean algebras and distributive lattices and H.Wallman[9] used lattice theoretic constructs to obtain the Wallman compactification .Mekinsey and Tarski[7] studied the " algebra of topology " that is topology studied from a Lattice theoretical viewpoint. But a fundamental change in the outlook came in late fifties; Charles Ehresmann[3]first articulated the view that a complete lattice with an appropriate distributivity property deserved to be studied in their own right rather than simply as a means to study topological spaces. He called the lattice a local lattice. Dowker and strauss[1] introduced the term frame for a local lattice and extended many results of topology to frame theory. It was with the publication of John Isbell,s [4] that the real importance of the subject emerged. Since then frame theory is 
studied extensively by many authors. The structure of the paper is as follows. In the second section is devoted to the basic definition and results concerning frames and fuzzy sets theory .

In section three the category FFrm of fuzzy frame and fuzzy frame homomorphism is defined. The main aim of section four is to show that there exist products and coproducts in the category FFrm and to construct them explicitly.

\section{Preliminaries}

Definition 2.1. A set $P$ equipped with a partial order $\leq(\leq$ is reflexive, transitive, antisymmetric) is called a partially ordered set, and usually called a poset for short.

Definition 2.2. A poset $P$ such that for any two $a, b \in P$ there is the infimum $a \wedge b$ and supremum $a \vee b$ is called a lattice.

Definition 2.3. A complete lattice is a poset in which each subset has an infimum and a supremum. In particular, a complete lattice has the bottomo and top1.

Definition 2.4. A lattice $L$ is distributive if

$$
\forall a, b, c \in L, \quad a \wedge(b \vee c)=(a \wedge b) \vee(a \wedge c)
$$

Which is equivalent to

$$
a \vee(b \wedge c)=(a \vee b) \wedge(a \vee c)
$$

A function $f: M \rightarrow N$ between two lattices is said to be increasing (decreasing) if

$$
f\left(m_{1}\right) \leq f\left(m_{2}\right) \text { Whenever } m_{1} \leq m_{2}\left(m_{1} \geq m_{2}\right) .
$$

Definition 2.5. A frame is a complete lattice $L$ satisfying the distributivity $\operatorname{Law}(\vee A) \wedge b=$ $\vee\{a \wedge b \mid a \in A\}$ for each subset $A \subseteq L$ and $b \in L$.

Definition2.6. A frame homomorphism $h: L \rightarrow M$ is a mapping preserving all suprema (including the bottom) and all finite intima (including the top1).

The category of frames and frame homomorphism will be denoted by Frm.

\section{Example 2.7.}

(i):Take $I$ an the unit interval,then $I=[0,1]$ is a frame.

(ii): For every topological space $(X, \tau), \tau$ is a frame.

Definition2.8. Let $X$ is a nonempty ordinary set, $L$ a complete lattice. An $L_{-}$fuzzy subset on $X$ is a mapping $A: X \rightarrow L$. 
Definition2.9. Let $L^{X}$ be an $L_{-}$fuzzy space. Define the partial order $\leq$in $L^{X}$ by:

$$
\forall A, B \in L^{X}, A \leq B \Leftrightarrow \forall x \in X, A(x) \leq B(x)
$$

Then $\left(L^{X}, \leq\right)$ is a poset.

Definition2.10.For $A, B \in L^{X}$ we define $A \vee B$ and $A \wedge B$ as :

$$
(A \vee B)(x)=A(x) \vee B(x), \quad(A \wedge B)(x)=A(x) \wedge B(x), \quad \forall x \in X .
$$

Thus, $\stackrel{o}{-}$ and $\underline{1} \in L^{X}, \underline{o}: X \rightarrow L$ such that $\stackrel{o}{-}(x)=o, \underline{1}(x)=1$ for all $x \in X$.

Definition2.11. Let $L^{X}, L^{Y}$ is $L_{-}$fuzzy spaces, $f: X \rightarrow Y$ an ordinary mapping. Based on $f: X \rightarrow Y$, define $L_{-}$fuzzy mapping $\vec{f}: L^{X} \rightarrow L^{Y}$ and its $L_{-}$fuzzy reverse mapping $f: L^{Y} \rightarrow L^{X}$,

$$
\begin{gathered}
\overleftarrow{f}(A)(y)=v\{A(x): x \in X, f(x)=y\}, \forall A \in L^{X}, y \in Y . \\
\overleftarrow{f}(B)(x)=B(f(x)), \forall A \in L^{Y}, x \in X .
\end{gathered}
$$

\section{Fuzzy Frame}

Definition 3.1.Let $L$ be a frame, then a fuzzy set $\{\mu: L \rightarrow I\}$ of $L$ is said to be a fuzzy frame if:

$\left(F_{1}\right) \mu(\vee S) \geq \inf \{\mu(a) \mid a \in S\}$ for every arbitrary $S \subseteq L$.

$\left(F_{2}\right) \mu(a \wedge b) \geq \min \{\mu(a), \mu(b)\}$ for all $a, b \in L$.

$\left(F_{3}\right) \mu\left(e_{L}\right)=\mu\left(0_{L}\right) \geq \mu(a)$ for all $a \in L$ where $e_{L}$ and $0_{L}$ are respectively the unit and zero element of the frame $L$.

Example 3.2.consider the set $R$, of real numbers with usual topology $\tau$, which is a frame. Let $\mu$ be a

Fuzzy set in $\tau$ defined by,

Then $\mu$ is a fuzzy frame of $\tau$.

$$
\mu(u)=\left\{\begin{array}{cc}
\frac{1}{2} \quad u=R, \emptyset \\
2 & u \neq R, \emptyset
\end{array} \text { Where } u \in \tau\right.
$$

Proposition 3. 3.If $\mu$ is a fuzzy frame of $L$ then $\mu_{t}=\{x \in L \mid \mu(x) \geq t\}$ is a subframe of $L$ for any $t \in I$ with $t \leq \mu\left(e_{L}\right)=\mu\left(o_{L}\right)$.

Proof. For arbitrary $\left\{a_{i}\right\}_{i \in \Lambda} \subseteq \mu_{t}$ we have $\mu\left(v a_{i}\right) \geq t$ since, $\mu$ is a fuzzy frame and $\mu\left(a_{i}\right) \geq t$ for all i . Hence $\vee a_{i} \in \mu_{t}$. Similarly for all $a, b \in \mu_{t}$ we have $a \wedge b \in \mu_{t}$ also clearly $e_{L}, o_{L} \in \mu_{t}$ therefore , $\mu_{t}$ is a subframe of $L$.

Proposition3. 4. If each non-empty level subset $\mu_{t}, t \in I$ of a fuzzy set $\mu$ is a subframe of $L$, then $\mu$ is a fuzzy frame of $L$.

Proof. Given $\mu_{t}=\{x \in L \mid \mu(x) \geq t\}, t \in I$ is a subframe of $L$. $\mu_{t}$ being a sub frame $o_{L}, e_{L} \in \mu_{t}, t \in I$. In particular we have $o_{L}, e_{L} \in \mu_{T}$ where $T$ the largest element of $I$ such that $\mu_{T} \neq \emptyset$.

Hence $\mu\left(e_{L}\right)=\mu\left(o_{L}\right)=T \geq \mu(a)$ for all $a \in L$, now let $S$ an arbitrary subset of $L$ and let $t=$ $\inf \{\mu(a) \mid a \in S\}$. Clearly we have $S \subseteq \mu_{t}$ hencev $S \in \mu_{t}$ and there for $\mu(\vee S) \geq \inf \{\mu(a) \mid a \in S\}$.

Similarly for all $a, b \in L$ we have $\mu(a \wedge b) \geq \min \{\mu(a), \mu(b)\}$.Hence $\mu$ is a fuzzy frame of $L$. 
Theorem 3.5. $\mu$ is a fuzzy frame of $L$ if and only if for any $\alpha \in I, \tau_{\alpha}=\{u \in L \mid \mu(u)>\alpha\}$ is a sub frame of $L$ for any $\alpha \in I$ with $\alpha<\mu\left(e_{L}\right)=\mu\left(0_{L}\right)$.

Proof. Follows from proposition 3.3 and proposition3.4.

Definition 3.6.If there are two fuzzy frame $\mu_{1}, \mu_{2}$ on the same frame $L$, we say that $\mu_{1}$ is stronger than $\mu_{2}$ if $\mu_{1}(a) \geq \mu_{2}(a)$ for every $a \in L$.

Definition3.7.Let $(L, \mu)$ be a fuzzy frame we define the mapping $\mu^{*}: L \rightarrow I$ by the equality $\mu^{*}(a)=$ $\mu\left(a^{c}\right)$ for every $a$ in $L$.The number $\mu^{*}(a)$ will be called the degree of complement of a fuzzy set $\mu$.

From the definition(3.1) and (3.6) one easily gets the following.

Proposition3.8.The mapping $\mu^{*}: L \rightarrow I$ has the following properties:

1) If $a, b \in L$, then $\mu^{*}(a \vee b) \geq \mu^{*}(a) \wedge \mu^{*}(b)$

3) $\mu^{*}(0)=\mu^{*}(1)=1$

Remark. It is clear that a fuzzy frame can be equivalently defined as a pair $\left(X, \mu^{*}\right)$ where $\mu^{*}: L \rightarrow I$ satisfies the properties $F_{1}, F_{2}, F_{3}$ and is understood as the degree of closeness of fuzzy subsets. the corresponding fuzzy frame in to be defined by the equality $\mu(a)=\mu^{*}\left(a^{c}\right)$.

Definition3.9. Let $(L, \mu)$ and $(M, \eta)$ be fuzzy frames and $f: L \rightarrow M$ is a frame homomorphism this mapping is called fuzzy continuous if $\mu\left(f^{-1}\right)(b) \geq \eta(b), b \in M$.

Proposition3.10.Let $\tilde{f}:(L, \mu) \rightarrow(M, \eta)$ and $\tilde{g}:(M, \eta) \rightarrow(N, \gamma)$ be morphism then $\tilde{g} o \tilde{f}:(L, \mu) \rightarrow$ $(N, \gamma)$ is a fuzzy frame homomorphismgof: $L \rightarrow M$ such that $\mu \geq$ rogof.

Proof. The proof is obvious.

Since the composition is associative and the identify mappinge: $L \rightarrow L$ is fuzzy frame homomorphism with respect to any fuzzy frame on $L$. The following definition is justified.

Definition3.11. By FFrm we denote the category the objects of which are fuzzy frames and the morphisms are fuzzy frame homomorphism between them.

\section{Products and coproducts of fuzzy frames}

The main aim of this section is to show that there exist products and coproducts in the category FFrm and to construct them explicitly. 


\subsection{The initial fuzzy frame for a mapping}

Let $L$ be a frame, $(M, \mu)$ a fuzzy frame and $f: L \rightarrow M$ is a frame homomorphism .By the initial fuzzy frame for this frame homomorphism we understand the weakest fuzzy frame $\tau$ on $L$ such that the mapping $f:(L, \tau) \rightarrow(M, \mu)$ is fuzzy frame homomorphism.

To construct such a fuzzy frame consider the set $S=\left\{b=f^{-1}(a) \mid a \in M\right\}$ of fuzzy subframes of $L$. for a given $b \in S$, Let $\rho_{b}=\left\{a \mid a \in M, b=f^{-1}(a)\right\}$ and define $\tau(b)=\sup \left\{\mu(a) \mid a \in \rho_{b}\right\}$. It is obvious that $\bigcup\left\{\rho_{b} \mid b \in L\right\}=M$ and $\tau\left(f^{-1}(a)\right) \geq \mu(a)$ for every $a \in M$.

Let $b_{1}, b_{2} \in S$, then $b=b_{1} \wedge b_{2} \in S$ and moreover, $\rho_{b} \subseteq\left\{a_{1} \wedge a_{2} \mid a_{1} \in \rho_{b_{1}}, a_{2} \in \rho_{b_{2}}\right\}$.

Therefore

$$
\begin{gathered}
\tau(b)=\sup \left\{\mu(a) \mid a \in \rho_{b}\right\} \geq \sup \left\{\sigma\left(a_{1} \wedge a_{2}\right) \mid a_{1} \in \rho_{b_{1}}, a_{2} \in \rho_{b_{2}}\right\} \geq \sup \left\{\mu\left(a_{1}\right) \wedge \mu\left(a_{2}\right) \mid a_{1} \in \rho_{b_{1}}, a_{2} \in\right. \\
\left.\rho_{b_{2}}\right\}=\sup \left\{\mu\left(a_{1}\right) \mid a_{1} \in \rho_{b_{1}}\right\} \wedge \sup \left\{\mu\left(a_{2}\right) \mid a_{2} \in \rho_{b_{2}}\right\}=\tau\left(b_{1}\right) \wedge \tau\left(b_{2}\right),
\end{gathered}
$$

and hence

$$
\tau(b) \geq \tau\left(b_{1}\right) \wedge \tau\left(b_{2}\right) \quad \text { for } b_{1}, b_{2} \in S
$$

In a similar way we can show that for any subfamily $\left\{b_{i} \mid i \in J\right\}$ of $S$

$$
\tau\left(v_{i} b_{i}\right) \geq \wedge_{i}\left\{\sup \mu\left(a_{i}\right) \mid a_{i} \in \rho_{b_{i}}\right\}=\wedge_{i} \tau\left(b_{i}\right)
$$

Moreover, it is obvious that $0=f^{-1}(0) \in S, 1=f^{-1}(1) \in S$ and $\tau(0)=\tau(1)=1$

Thus $\tau: S \rightarrow I$ satisfies the axioms of definition(3.1).Now we extend $\tau$ to a mapping $\tau: L \rightarrow I$ by letting $\tau(b)=0$ for allb $\notin S$.

It is easy to cheak that the function $\tau$ thus defined is indeed a fuzzyframe.Moreover, from the construction it is clear that $\tau$ is the weakest fuzzy frame on $L$ making the mapping $f:(L, \tau) \rightarrow(M, \mu)$ fuzzy frame homomorphism.

\subsection{Initial fuzzy frame for a family of mappings}

Let now $\left.\left\{M_{\alpha}, \mu_{\alpha}\right) \mid \alpha \in A\right\}$ be a family of fuzzy frame and consider for each $\alpha \in A$ a mapping $f_{\alpha}: L \rightarrow M_{\alpha}$. Let $\tau_{\alpha}: L \rightarrow I$ be the initial fuzzy frame on $L$ for $f_{\alpha}$ and let the mapping $\tau: L \rightarrow I$ be defined by the equality $\tau(b)=i n f_{\alpha} \tau_{\alpha}(b)$ where $b \in L$. Since $\tau\left(b_{1} \wedge b_{2}\right)=\inf f_{\alpha} \tau_{\alpha}\left(b_{1} \wedge b_{2}\right) \geq i n f_{\alpha}\left(\tau_{\alpha}\left(b_{1}\right) \wedge \tau_{\alpha}\left(b_{2}\right)\right) \geq$ $\inf f_{\alpha} \tau_{\alpha}\left(b_{1}\right) \wedge i n f_{\alpha} \tau_{\alpha}\left(b_{2}\right)=\tau\left(b_{1}\right) \wedge \tau\left(b_{2}\right)$ and $\tau\left(v_{i} b_{i}\right)=i n f_{\alpha} \tau_{\alpha}\left(v_{i} b_{i}\right) \geq i n f_{\alpha} \wedge_{i} \tau_{\alpha}\left(b_{i}\right)=\wedge_{i} i n f_{\alpha} \tau_{\alpha}\left(b_{i}\right)=$ $\wedge_{i} \tau\left(b_{i}\right)$.

for any collection of fuzzy subset $b_{i}$ of $L$, one can easily conclude that $\tau$ is a fuzzy frame on $L$.Moreover ,it is clear from (4.1) and from the construction of $\tau$ that it is the weakest fuzzy frame on $L$ for which mappings $f_{\alpha}:(L, \tau) \rightarrow\left(M_{\alpha}, \mu_{\alpha}\right)$ are fuzzy continuous. This fuzzy frame $\tau$ will be called the intial fuzzy frame for the family of mapping $\left\{f_{\alpha}: L \rightarrow M_{\alpha} \mid \alpha \in A\right\}$.

The existence of such a fuzzy frame allows us to state the following theorem: 
Theorem4.2.1 . FFrm is a complete category . In particular, FFrm contains products and inverse limits.

\subsection{Product of fuzzy frames}

To construct the product in FFrm explicitly considers a family $\left\{\left(L_{\alpha}, \tau_{\alpha}\right) \mid \alpha \in A\right\}$ of fuzzy frames. The product of this family can be defined as a pair $(L, \tau)$, where $L$ denotes the product of all sets $L_{\alpha}$ and $\tau$ is the initial fuzzy frame generated on $L$ by the family $\left\{\rho_{\alpha}: L \rightarrow L_{\alpha}, \alpha \in A\right\}$ of all projections.

Let $\left(L_{1}, \tau_{1}\right),\left(L_{2}, \tau_{2}\right)$ be two fuzzy frame and let $(L, \tau)$ denote their product. If $b_{1} \in L_{1}, b_{2} \in L_{2}$ then for $b=b_{1} \times b_{2} \in L$ which is defined as $b=b_{1} \wedge b_{2}$ we have

$$
\tau(b)=\tau\left(\rho_{1}^{-1}\left(b_{1}\right) \wedge \rho_{2}^{-1}\left(b_{2}\right)\right) \geq \tau\left(\rho_{1}^{-1}\left(b_{1}\right)\right) \wedge \tau\left(\rho_{2}^{-1}\left(b_{2}\right)\right) \geq \tau_{1}\left(b_{1}\right) \wedge \tau_{2}\left(b_{2}\right) .
$$

Hence the degree of openess of the product of two fuzzy sets in the product space is not less than the minimal dgree of openess of these sets in the corresponding fuzzy spaces.

Now let $\left\{\left(L_{\alpha}, \tau_{\alpha}\right) \mid \alpha \in A\right\}$ be a family of fuzzy frames and let $(L, \tau)$ denote their product. Take $b_{\alpha} \in L_{\alpha}$ for every $\alpha$ and let $b \in L$ denote the product of all $b_{\alpha}\left(\right.$ i.e $\left.b=\wedge_{\alpha} \mu_{\alpha}\left(b_{\alpha}\right)\right)$. Quite similarly as above one can show that $\tau^{*}(b) \geq \wedge \tau^{*}{ }_{\alpha}\left(b_{\alpha}\right)$, and hence the degree of closednees of the product of fuzzy sets in not less than the degree of the degree of closeness of the factors.

The rest of this section is devoted to the concept of coproduct (or direct sum) of fuzzy frames and to some closely related notions.

\subsection{Final fuzzy frame for a mapping}

Let $(L, \tau)$ be a fuzzy frame and $M$ is a set consider a mapping $f: L \rightarrow M$ and for every $a \in M$ let $\mu(a)=$ $\tau\left(f^{-1}(a)\right)$.

It is easy to check that $\mu$ is a fuzzy frame on $M$ and more over, it is the strongest fuzzy frame on $M$ for which the mapping $f:(L, \tau) \rightarrow(M, \mu)$ is fuzzy frame homomorphism.

\subsection{Final fuzzy frame for a family of mappings}

Let $\left\{\left(L_{\alpha}, \tau_{\alpha}\right) \mid \alpha \in A\right\}$ be a family of fuzzy frames and for every mapping $f_{\alpha}: L_{\alpha} \rightarrow M$ where $M$ is a set . Let $\mu_{\alpha}$ denote the final topology on $M$ for $f_{\alpha}$. define $\mu: M \rightarrow I$ by the equality $\mu(a)=i n f_{\alpha} \mu_{\alpha}(\alpha)$ for $a \in$ $M$.

Quite similarity as in (4.2) one can show that $\mu$ is a fuzzy frame on $M$.More over, it is easy to notice, that it is the strangest fuzzy frame on $M$ for which all the mappings $f_{\alpha}: L_{\alpha} \rightarrow M$ are fuzzy frame continuous .

From (4.5) immediately follows such a theorem :

Theorem 4.5.1 . The category FFrm is cocomplete specifically, it contains coproducts and direct limits. 


\subsection{Coproduct in FFrm}

To construct the coproduct in FFrm explicity consider a family $\left\{L_{\alpha}, \tau_{\alpha} \mid \alpha \in A\right\}$ of fuzzy frames and let $L=\oplus L_{\alpha}$ denote the direct sum of the corresponding sets. The fuzzy frame $(L, \tau)$ where $\tau$ is the final topology for the family of all inclusions $i_{\alpha}: L_{\alpha} \rightarrow L$ is just the coproduct of these fuzzy frame.More over, it is easy to notice, that $\tau(b)=i n f_{\alpha} \tau_{\alpha}\left(b_{\alpha}\right)$ and $\tau^{*}(b)=\inf \tau_{\alpha}{ }^{*}\left(b_{\alpha}\right)$ where $\alpha$ denotes the restriction of $b \in L$ to $L_{\alpha}$.

\section{Acknowledgements}

This study was supported by Payamenoor University Research Grant.

\section{References}

[1] C.H. Dowker, D.Strauss, "Sums in the category of frames", J. Math. 3 (1977)7-15.

[2] J. Gutiérrez Garcia, U. Hohle, M.A, "de Prada Vicente, on lattice-valued frames: the completely distributive case", Fuzzy Sets and Systems. 161(2010)1022-1030.

[3] C.Ehresmann,"Gattungen von lokalen Strukturen", Jber. Deutsch. Math. Verein, 60(1957)59-77.

[4] J. Isbell, “Atomless parts of spaces", Math. Scand. 31(1972)5-32.

[5] J. Isbell, "Product spaces in locales", Proc, Amer. Math. Soc. (1) 81(1981)116-118.

[6] P.T. Johnstone, "Stone Spaces", Cambridge Studies in Advanced Mathematics 3, Cambridge University Press, (1982).

[7] J. C. C. Mckinsey, A.Tarski, “The algebra of topology”, Ann. Math. J. (2)45 (1944) 141-191.

[8] B. A. Mohideen, A. Ibrahim, "Fuzzy prime and anti-fuzzy prime implicative filters of lattice Wajsberg algebras", Journal of Mathematics and Computer Science, 9(2014)25-32.

[9] A.Pultr, S.E., "Rodabaugh,Lattice-valued frames.functor categories and classes of sober spaces, in Mathematics of fuzzy sets". Logic. Topology, and Measure Theory,U. Hohle and S.E. Rodabaugh (Eds.). The Handbooks of Fuzzy sets series: Vol. 3, Kluwer Academic Publishers Dordrecht (1999), pp.153-187.

[10] T. Rajesh, "A Study of Frames in the Fuzzy and Intuitionistic Fuzzy Contexts", Ph.D Thesis, Cochin University of Science and Technology, Kerala (2006).

[11] M. H. Stone, "The theory of representation for Boolean algebras", Trans. Amer. Math. Soc. 40 (1936) 37-111.

[12] H. Wallman, "Lattices and topological spaces", Ann. Math. (2) 39 (1938) 112-26. Z18-332.

[13] S. Willard, "General topology", Addison-Wesley Publishing Company, (1970).

[14] W. Yao, “An approach to fuzzy frames via fuzzy posets”, Fuzzy Sets and Systems.166 (2011) 75 $-89$ 